\title{
Erratum: Prospects for detection and application of the alignment of galaxies with the large-scale velocity field [Phys. Rev. D 102, 123507 (2020)]
}

\author{
I. R. van Gemeren and N. E. Chisari॰
}

(Received 12 August 2021; published 8 September 2021)

DOI: 10.1103/PhysRevD.104.069902

We have identified two errors in the implementation of the forecast in our previous work which affect the results in Table 11 and the error bars shown in Fig. 1 for the velocity-intrinsic shape power spectrum. These do not change the qualitative conclusions of the work, but result in overall lower significance for the signal investigated.

First, in Eq. (22) the noise of the velocity power spectrum was implemented by multiplying the velocity noise spectrum by $\mu^{-2}$, where $\mu$ is the angle with respect to the line of sight. This factor of $\mu^{-2}$ has to be removed as we are only interested in correlating the intrinsic shape field with the radial part of the velocity field. Second, we found a bug in the implementation of the velocity noise power spectrum, which has now increased.

Table 11 should be replaced by Table I, where the main change is a decrease in the signal-to-noise for the radial velocityintrinsic shape dipole. $P_{\hat{v}_{r} E}^{(1)}$ is less sensitive to changes in $k_{\max }$, as the signal-to-noise saturates more quickly than in our previous results. We show a new version of Fig. 1.

There is also a slight change in the value of the scale dependence parameter $\alpha$ in Eq. (32). For the velocity shape correlation, this value becomes $\alpha=0.026$. The value for the galaxy shape correlation does not change. The conclusion of Sec. V B remains unchanged. However we do not see a gain in $\mathrm{S} / \mathrm{N}$ anymore with increasing $k_{\max }$, so there is no sign that exploring in the nonlinear regime would be valuable.

Lastly, we document a small number of typos:

(i) in Table I, the comoving volume of the survey should be $0.10 \times 10^{11} \mathrm{Mpc}^{3}$;

(ii) in Eqs. (1), (12) and (13) it should be respectively $v(\mathbf{k}) \propto\left(\mathbf{k} / k^{2}\right) \delta(\mathbf{k}), \quad v(\mathbf{k})=\frac{f a H}{k} \delta(\mathbf{k}) \quad$ and $v_{r}(\mathbf{k})=\frac{i k_{r}}{k} v(\mathbf{k})=\mu \frac{f a H}{k} \delta(\mathbf{k})$

(iii) the redshift-space distortion factor should be $\left(1+f \mu^{2} / b_{g}\right)$ in Eq. (16).

None of these affect the results of the study. As the $\mathrm{S} / \mathrm{N}$ values for the velocity shape power spectrum are still significant our conclusions remain qualitatively unchanged.

TABLE I. Updated results of the Fisher forecast for the parameter $\tilde{C}_{1}$.

\begin{tabular}{lccc}
\hline \hline Correlation & $k_{\max }\left[\mathrm{Mpc}^{-1}\right]$ & $\delta \tilde{C}_{1} / \tilde{C}_{1}$ & $\mathrm{~S} / \mathrm{N}$ ratio \\
\hline$P_{\hat{v}_{r} E}^{(1)}$ & 0.2 & 0.19 & 7.4 \\
& 0.5 & 0.19 & 7.4 \\
& 1 & 0.19 & 7.4 \\
$P_{g E}^{(0)}$ & 0.2 & 0.032 & 44 \\
& 0.5 & 0.023 & 62 \\
& 1 & 0.021 & 69 \\
\hline \hline
\end{tabular}




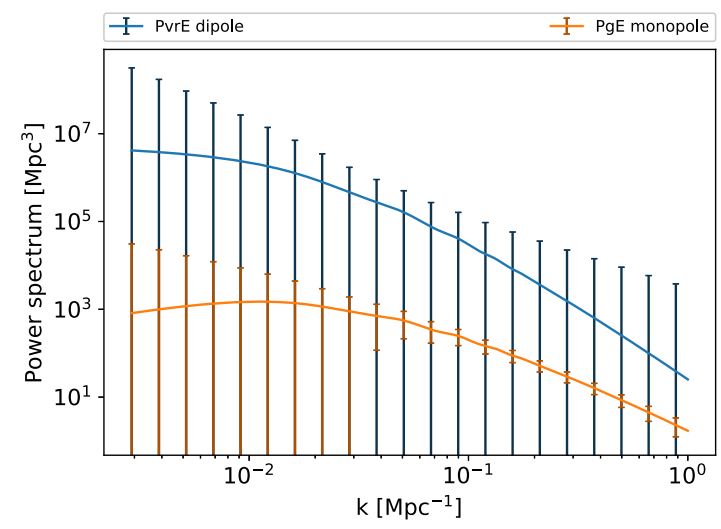

FIG. 1. Dipole $P_{\hat{v}_{r} E}$ (blue) and monopole $P_{g E}$ (orange) as a function of wave number for a value of $k_{\max }=1 \mathrm{Mpc}{ }^{-1}$, with accompanying error bars corresponding to the 4MOST + LSST combination.

We thank Karel Zwetsloot for pointing out some of these issues. This work is part of the Delta ITP consortium, a program of the Netherlands Organisation for Scientific Research (NWO) that is funded by the Dutch Ministry of Education, Culture and Science $(\mathrm{OCW})$. 